\title{
Validation study of a conventional enzyme immunoassay to detect HIV antibodies in oral fluid
}

\author{
Stanekova $\mathrm{D}^{1}$, Mirandola $\mathrm{M}^{2,3}$, Gios $\mathrm{L}^{3}$, Botsi $\mathrm{C}^{4}$, Habekova $\mathrm{M}^{1}$, Gonzales-Soler $\mathrm{V}^{5,6,7}$, \\ Nikolopoulos $\mathrm{GK}^{4}$
}

Slovak Medical University, NRC for HIV/AIDS prevention, Bratislava, Slovakia. danica.stanekova@szu.sk

\begin{abstract}
OBJECTIVES: The aim of our study was to validate the Genscreen HIV $1 / 2$ version 2 (BIO-RAD) for detecting HIV antibodies in oral fluid specimens (OF).

BACKGROUND: The advantage of assays to detect HIV infection in OF lies in the on-site easy access and noninvasive sample collection.

METHODS: Paired serum and OF were collected from 496 subjects (263 HIV-positive and 233 HIV-negative) using the Oracol test kit (Oracle Diagnostics, Inc). The quality of OF was verified by measuring total IgGs using the Human IgG ELISA Quantitation Kit (Bethyl Lab.inc). All reactive OF samples were retested by Western blot HIV1/2 BLOT 2.2 (MP Biomedical, Singapore, China).

RESULTS: Of 263 OF samples from participants with blood-based HIV-positive results, 259 were positive by Genscreen HIV $1 / 2$ version 2 (98.48\% sensitivity, 95\% Cl; 96.2-99.6). The 233 individuals who had a non-reactive HIV blood test were found negative on testing their OF by Genscreen HIV $1 / 2$ version 2 (100\% specificity, 95\% Cl; 98.4-100). NPV and PPV of the assay were $98.31 \%(95 \% \mathrm{Cl}$; 95.74-99.34) and 100\%, $(95 \% \mathrm{Cl}$; 98.53-100.00), respectively.

CONCLUSION: Genscreen HIV $1 / 2$ version 2 (Bio-Rad) is a prospective method for HIV surveillance studies in hard-to-reach populations with high risk behavior using non-invasive OF collection (Tab. 1, Fig. 1, Ref. 16). Text in PDF www.elis.sk.

KEY WORDS: HIV testing, oral fluid, Genscreen HIV $1 / 2$ version 2.
\end{abstract}

\section{Introduction}

Many persons with HIV do not get tested until late in their infection (1). It is estimated that approximately $30-50 \%$ of HIV seropositive cases in European settings are diagnosed with Acquired Immune Deficiency Syndrome (AIDS) within a calendar

\footnotetext{
${ }^{1}$ Slovak Medical University, Slovakia, ${ }^{2}$ Infectious Diseases Section, Department of Pathology, Verona University Hospital, Verona, Italy, ${ }^{3} \mathrm{CReMPE}$ Regional Coordination Centre for European Project Management - Veneto Region, Verona University Hospital, Verona, Italy, ${ }^{4} \mathrm{Hellenic}$ Centre for Disease Control and Prevention, Athens, Greece, ${ }^{5} \mathrm{Centre}$ for Epidemiological Studies on HIV/STI in Catalonia (CEEISCAT) - ICO, Badalona, Barcelona, Spain, ${ }^{6}$ Department of Microbiology, Fundació Institut d'Investigació en Ciències de la Salut Germans Trias i Pujol, Hospital Universitari Germans Trias i Pujol, Universitat Autònoma de Barcelona, Badalona, Spain, and ${ }^{7}$ CIBER Epidemiología y Salud Pública (CIBERESP), Barcelona, Spain

Address for correspondence: D. Stanekova, NRC for HIV/AIDS prevention, Slovak Medical University, Limbova 14, SK-83301 Bratislava, Slovakia. Phone: +421.2.59370383, Fax: +421.2.59370869

Acknowledgements: The capacity to build estimation of HIV/ Syphilis prevalence by using non-invasive methods among MSM in Southern and Eastern Europe - SIALON project was funded by the European Commission under the European Commission Public Health Programme 20032008. We thank Dunia Ramarli from Immunology - Verona University Hospital, Italy for her useful remarks in the publication.
}

year of their diagnosis or have the CD4-cell count lower than 350/ $\mathrm{mm}^{3}$ at presentation $(2,3)$. In addition, a significant proportion of tested individuals fail to return to get their results (4).

Oral fluid (OF)-based HIV tests could promote early diagnosis and increase the number of people who become aware of their status. The major advantages of replacing serum/plasma with OF include easy access to testing and noninvasive collection while maintaining high-level performance $(5,6,7)$. OF tests do not require the presence of experienced healthcare personnel. Nevertheless, the staff should be trained on how to perform their assigned tasks $(5,7)$. In addition, OF-based testing is a key tool for successful implementation of Second Generation Surveillance approaches (8). The sampling methodologies for this purpose require collection of specimens from hard-to-reach populations at locations such as streets, bars, discos, and saunas where drawing blood is problematic and maybe unsafe. A CE marked OF-based assay could help collect and process easily, quickly and safely many samples in these settings.

HIV tests on OF had high levels of sensitivity and specificity across various populations and settings $(9,10,11,12)$. A recent meta-analysis of the accuracy of a rapid HIV-antibody-based pointof-care test (Oraquick advance rapid HIV-1/2) calculated pooled estimates of sensitivity and specificity at $98.03 \%$ and $99.74 \%$, respectively (13). 
Given the great public health interest in OF-based HIV tests, we decided to evaluate the performance of Genscreen HIV $1 / 2$ version 2 (Bio-Rad) on OF samples. This conventional assay is commercially available for use in serum or plasma. Preliminary results of this validation have been presented (14) as part of the SIALON I study that was conducted on men who have sex with men (MSM).

\section{Material and methods}

Paired serum and oral fluid specimens were collected from 496 subjects in 6 countries that participated in the European Union (EU)-funded project SIALON I (14). All study subjects gave informed consent to participate in the study. The HIV-positive group $(\mathrm{n}=263)$ included Western Blot (WB)-confirmed patients (based on blood specimens) who were attending healthcare facilities and were invited to take part in the study (regardless of whether being on antiretroviral treatment or not). Controls $(n=233)$ were randomly selected health professionals and volunteers who looked for HIV testing and were HIV-negatives according to Enzyme Immunoassay (EIA; based on blood specimens).

The collection and transportation of serum were done according to standard procedures. The Oracol test kit (Malvern Medicals, Worcester, UK) was used for OF collection. Oral fluid samples were kept in a fridge and sent to the laboratory within 72 hours. On receipt, the testing laboratory extracted OF from each swab adding $2.0 \mathrm{ml}$ of transport medium followed by vortex for 20-30 seconds. The transport medium was allowed to equilibrate with OF for at least 1 hour at $4{ }^{\circ} \mathrm{C}$, after which the swab was removed from the plastic tube. Following centrifugation, the OF was collected and filtered through a Celtron 30, 0.2- $\mu \mathrm{m}$ filter (Schleicher \& Schuell MicroScience, Dassel, Germany). The oral fluid was then stored in aliquots and at $-20^{\circ} \mathrm{C}$.

The quality of OF samples was evaluated and verified by measuring total IgGs using the Human IgG ELISA Quantitation Kit (Bethyl Laboratories. Inc). Oral fluid samples with $\operatorname{IgG}<3.5$ $\mathrm{mg} / \mathrm{l}$ were excluded from the study.

In this work, we evaluated Genscreen HIV $1 / 2$ version 2 that is produced by Bio-Rad and has received CE marking (conformation with European standards) for testing HIV antibodies in serum and plasma (HIV-1:100 \% sensitivity and $99.8 \%$ specificity; HIV-2: $100 \%$ sensitivity). The validation protocol we followed was developed according to the EU general principles set out in EU Commission Decision as of May 7th, 2002 on common technical specifications for in vitro diagnostic medical devices and the international guidelines for using HIV testing technologies in surveillance (WHO \& UNAIDS, 2009) .

All reactive OF samples were retested by Western blot HIV1/2 BLOT 2.2 ( MP Biomedical, Singapore, China) according to standard procedures described in the instructions package for serum/ plasma testing.

\section{Results}

Of 263 oral fluid specimens from participants with bloodbased EIA-reactive and WB-confirmed results, 259 were as well
Tab. 1. Results of evaluating Genscreen HIV 1/2 version 2 (Bio-Rad) on oral fluid vs. serum samples.

\begin{tabular}{lccccc}
\hline Oral fluid/serum & $\begin{array}{c}\text { HIV } \\
\text { negative }\end{array}$ & $\begin{array}{c}\text { HIV } \\
\text { positive }\end{array}$ & Total & $\%$ & \\
\hline HIV negative & 233 & 4 & 237 & 98.31 & NPV \\
HIV positive & 0 & 259 & 259 & 100.00 & PPV \\
Total & 233 & 263 & 496 & & \\
$\%$ & 100.00 & 98.48 & & & \\
& specificity & sensitivity & & & \\
\hline
\end{tabular}

positive by Genscreen HIV $1 / 2$ version 2 . This corresponds to an overall sensitivity of $98.48 \%(95 \% \mathrm{CI} ; 96.2-99.6)$. The $233 \mathrm{OF}$ samples of the blood-based EIA-negative individuals were correctly identified as negative cases by Genscreen HIV $1 / 2$ version (100\% specificity, 95\% CI; 98.4-100). The Negative Predictive Value (NPV) and the Positive Predictive Value (PPV) of the assay were $98.31 \%$ (95\% CI; 95.74-99.34) and $100 \%$ (95\% CI; 98.53100.00), respectively (Tab. 1). Assuming an HIV prevalence of 5 $\%$ and $15 \%$, the NPVs were $98.5 \%$ and $99.73 \%$, respectively.

Three of the four oral fluid samples that were falsely negative by Genscreen HIV $1 / 2$ version 2 were positive in Western blotting (Fig. 1) while 1 oral fluid sample showed one reactive band for HIV-1 (gp160), by using a five-fold amount of regular loading compared to serum testing (100 microlitres instead of 20).

\section{Discussion}

HIV testing on oral fluid has fewer requirements in terms of local staff training, it reduces the risk of injury from sharp instruments while it increases clients' comfort and acceptability (7). Given the multiple advantages of OF-based sampling and testing, we evaluated the performance of the conventional Genscreen HIV $1 / 2$ version 2 (Bio-Rad) assay by testing paired oral fluid and serum samples collected from 496 subjects in $6 \mathrm{EU}$ countries that had

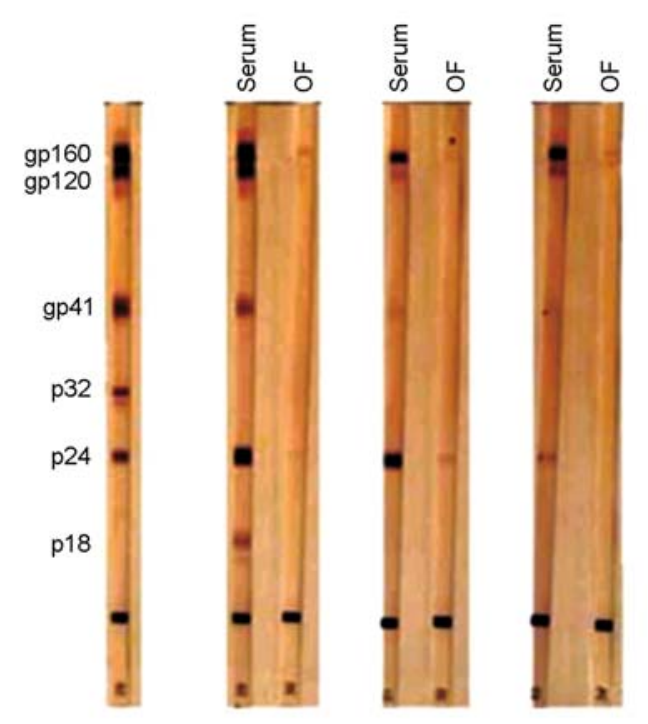

Fig. 1. Positive Western Blots of 3 false EIA-negative oral fluid samples. 
participated in the SIALON I project (14). The assay performed very well at a sensitivity of $98.48 \%$ and specificity of $100 \%$. Our results are comparable with the previous research that also showed high-level performance on oral fluids of both Genscreen HIV $1 / 2$ vs 2 (Bio-Rad) (10) and fourth generation Genscreen assay, the Gensceen Ultra HIV Ag-Ab (5).

In our study, 3 of 4 OF samples that were false negatives by Genscreen HIV $1 / 2$ version 2 assay were found positive in Western Blot testing. The other falsely negative oral fluid sample had a reactive band for HIV-1 (gp160) when we used a five-fold amount of regular loading compared to serum testing. The false OF-based negativity could be due to low levels of functional IgGs as a result of IgG degradation, bacterial contamination, or erroneous storage. Although the OF-based tests are simple and reliable, mistakes can occur at any point of the process. To reduce errors, a quality assurance program should be in place.

Recent research has also questioned the accuracy of oral fluidbased tests (in terms of sensitivity), especially when used soon after infection $(15,16)$. A meta-analysis in particular found a 2 $\%$ lower sensitivity of an OF-based test compared to blood-based analyses (13). In our study, however, we lacked the information for assessing the performance of the Genscreen HIV $1 / 2$ version 2 (Bio-Rad) on samples from recently infected individuals, and especially whether the 4 false negatives were derived from patients who had acquired HIV recently. OF-based tests might need further evaluation in the setting of acute and recent HIV infection.

\section{Conclusions}

The conventional Genscreen HIV $1 / 2$ version 2 (Bio-Rad) assay shows high-level performance for the diagnosis of HIV infection on oral fluids. It could thus be a suitable tool for HIV testing of oral fluids accessible to people in both clinical and non-clinical settings. Additionally, it could also prospectively contribute to enhancing the uptake of testing and implementing Second Generation HIV Surveillance in hard-to-reach populations with high-risk behavior.

\section{References}

1. ECDC/WHO. HIV/AIDS surveillance in Europe 2012. 2013; ECDC.

2. Janiec J, Haar K, Spiteri G, Likatavicius G, Van de Laar M, Amato-Gauci AJ. Surveillance of human immunodeficiency virus suggests that younger men who have sex with men are at higher risk of infection, European Union, 2003 to 2012. Euro Surveillance: Bulletin Européen Sur Les Maladies Transmissibles $=$ European Communicable Disease Bulletin. 2013; 18: 20644.
3. Wilson K d'Almeida, Dray-Spira R, Aubrière C, Hamelin C, Spire B, Lert, F. Frequency and correlates of late presentation for HIV infection in France: older adults are a risk group - results from the ANRS-VESPA2 Study, France. AIDS Care 2014; 26: S83-93.

4. Cabié A, Bissuel F, Huc P, Paturel L, Abel S. Impact of rapid HIV testing on the return rate for routine test results in sexually transmitted infection testing centres. Internat J STD AIDS 2011; 22: 757-758.

5. Rayment M, Doku E, Thornton A et al. Automatic oral fluid-based HIV testing in HIV screening programmes: automatic for the people. HIV Med 2013; 3: 49-52.

6. Semá Baltazar C, Raposo C, Jani IV, Shodell D et al. Evaluation of the performance and acceptability of two oral fluid HIV rapid tests in Mozambique. J Clin Microbiol 2014; 52: 3544-3548.

7. WHO, \& UNAIDS. Guidelines for Using HIV Testing Technologies in Surveillance. Geneva, 2009.

8. WHO, \& UNAIDS. Guidelenes for second generation HIV surveillance: an Update: Know your epidemic. Geneva, 2013.

9. Delaney KP, Branson BM, Uniyal A et al. Performance of an oral fluid rapid HIV-1/2 test: experience from four CDC studies. AIDS 2006; 20: $1655-1660$.

10. Fransen K, Vermoesen T, Beelaert G et al. Using Conventional HIV tests for HIV Diagnosis on Oral Fluid. J Virol Methods 2013; 194: 46-51.

11. Stanekova D, Habekova M, Wimmerova S, Gramblickova I. HIV infection and sexual behaviour among homosexuals and bisexual men in Bratislava. Cent Eur J Publ Health 2000; 8: 172-175.

12. Zelin J, Garrett N, Saunders J et al. North East London Sexual Health Network Research Consortium. An evaluation of the performance of OraQuick ADVANCE Rapid HIV-1/2 Test in a high-risk population attending genitourinary medicine clinics in East London, UK. Int J STD AIDS 2008; 19: 665-667.

13. Pant Pai N, Balram B, Shivkumar S et al. Head-to-head comparison of accuracy of a rapid point-of-care HIV test with oral versus wholeblood specimens: a systematic review and meta-analysis. Lancet Infect Dis 2012; 12 : 373-380.

14. Mirandola M, Folch Toda C, Krampac I et al. HIV bio-behavioural survey among men who have sex with men in Barcelona, Bratislava, Bucharest, Ljubljana, Prague and Verona, 2008-2009. Eurosurveillance 2009; 14: 48 .

15. Pilcher CD, Louie B, Facente $S$ et al. Performance of rapid point-ofcare and laboratory tests for acute and established HIV infection in San Francisco. PLoS One 2013; 8: 12.

16. Luo W, Masciotra S, Delaney KP, Charurat $M$ et al. Comparison of HIV oral fluid and plasma antibody results during early infection in a longitudinal Nigerian cohort. J Clin Virol 2013; 58: 1.

Received April 7, 2015. Accepted June 26, 2015. 